\title{
Electrochemical signature of hen egg white lysozyme at the glycerol- modified liquid-liquid interface
}

Benjamin J. J. Austen and Damien W. M. Arrigan*

Nanochemistry Research Institute \& Department of Chemistry, Curtin University, GPO Box U1987, Perth, Western Australia, 6845, Australia

* Author for correspondence. Email d.arrigan@curtin.edu.au

\begin{abstract}
Electrochemical characterization of hen egg white lysozyme (HEWL) at a glycerolmodified interface between two immiscible electrolyte solutions (ITIES) was conducted using a microporous silicon membrane-supported gelled-1,6dichlorohexane/water-glycerol interface. The electrochemical response of HEWL under these conditions is of interest for the system's potential application to the formation of isorefractive emulsified-ITIES, which offer practical opportunities for spectrophotometric analysis of interfacial processes. Importantly, the voltammetric signature for HEWL seen under glycerol-rich conditions was similar but with some differences from that for glycerol-free conditions. Specifically, the potential at which facilitated transfer of the organic phase electrolyte anion tetrakis-(4chlorophenyl) borate ( $\mathrm{TPBCl}^{-}$) occurred was shifted to lower potential with increasing glycerol concentration. However, features in the voltammetry associated with adsorption/desorption processes were observed to remain constant. The simple ion transfer response of tetraethylammonium cations $\left(\mathrm{TEA}^{+}\right)$at the same glycerolmodified ITIES provides insight into the nature of changes that determine the atypical HEWL signature. Lower ion transfer current with respect to increasing glycerol concentration and a shift in transfer potential were the key findings here. The results indicate that the electrochemistry which determines the HEWL signature is similar in environments that are rich in glycerol or purely aqueous.
\end{abstract}


ITIES; interface; lysozyme; glycerol-modified; voltammetry

\section{Introduction}

Voltammetry at the interface between two immiscible electrolyte solutions (ITIES) provides a platform for the non-redox detection of analytes in a range of solution environments[1]. This system has had particular success applied to quantitative analysis of biomacromolecules[2], where the detection mechanism is suggested to involve facilitated ion transfer of the organic phase electrolyte anion by a cationic protein, and adsorption of this protein-anion complex on the interface[3]. There has been a significant amount of work directed towards understanding the behaviour of proteins at the ITIES, with a view to improving detection selectivity for medically and biologically important species. In a more general sense, a better understanding of the way proteins and other large biomolecules interact with polarised liquid-liquid interfaces is also important for drug delivery applications[4] as well as study into biological systems[5]. Voltammetric studies have traditionally been the preferred tool for investigating these systems[2], however in recent years novel mass spectrometry techniques have broken new ground in elucidating the structure of these analytes at the polarised interface[6,7]. To date, studies employing such techniques have delivered insight into the interfacially-mediated formation of protein-anion complexes[7] and protein tertiary structure, with respect to interfacial proximity and applied potential[6]. There are many well established techniques for characterising protein structure; spectrophotometric techniques such as Fourier transform infra-red (FTIR)[8] and circular dichroism (CD) spectroscopies [9] are of particular interest. It is, however, difficult to characterise proteins within an ITIES system as the geometry of the system, often at the microor nanoscale, prohibits the use of regular laboratory spectrophotometric systems. In addition, the characterisation method must not significantly affect the physical environment within which the protein analyte resides, and upon which the interfacial environment is dependent. The studies reported in this paper concern voltammetry under glycerol-rich conditions which represent those required to implement an in situ spectrophotometric analysis of the polarised ITIES.

The recently-established refractive index matched emulsion (RIME) $[10,11]$ system consists of an organic phase dispersed in a glycerol-water aqueous phase, the 
proportions of which render the emulsion isorefractive. It follows that an emulsion stabilised by addition of a surface active protein (Pickering emulsion), such as hen egg white lysozyme (HEWL), may then be subjected to bulk phase spectrophotometric measurements, such as CD or FTIR, in order to characterise the protein structure as a function of various parameters, e.g. interfacial potential, solvent composition, $\mathrm{pH}$, etc.

To implement the refractive index matched ITIES, it is necessary to modify the character of one or both phases. A surprisingly limited body of work has dealt with modification of the aqueous phase of the ITIES. Previously, frozen[12] and gelled aqueous phases[13-15] have been employed and in these cases, the aim has been to modify the mechanical properties of the aqueous phase. Indeed, gelling of the aqueous phase by addition of a polymer, such as agar, agarose, $\mathrm{k}$-carrageenan or chitosan, is commonly employed to modulate aqueous phase diffusion processes [15], but no work has reported using glycerol as an additive, despite the clear potential for probing the chemically-dependent nature of interfacial interactions and analyte conformation. Of course, the chemical composition of the phases defines not only the solvation energy, and consequently the potential at which processes occur (Gibbs energy of transfer), but also the analyte's structure. The latter may be especially important in the case of large bio-macromolecules, which of ten exhibit a structural dependence on their solvation environment that is more significant, in terms of molecular activity and function, than typically observed for much smaller molecules. HEWL exhibits some dependence on glycerol-water solvent system composition[16], which is important to note as it also exhibits complex and structurally dependent interactions with the $\operatorname{ITIES}[3,6,7,17]$. By contrast, any solvation dependence exhibited by small analyte ions, such as tetraethyl ammonium cations $\left(\mathrm{TEA}^{+}\right)$, ought to result in comparatively insignificant changes to the electrochemical response, as the ion transfer (IT) mechanism that small tetraalkylammonium ions undergo is expected to be insensitive to solvent-induced conformational change. It is therefore interesting to compare the voltammetric responses of HEWL and $\mathrm{TEA}^{+}$in discussing glycerol-modification of the ITIES.

HEWL is a model bio-macromolecular polyionic analyte, chosen for its surface activity, tolerance to relatively low $\mathrm{pH}$, and high denaturation temperature (ca. $74.8^{\circ} \mathrm{C}[18]$ ). Previous studies employing HEWL as an analyte at the ITIES proposed 
three mechanistic features: facilitated ion-transfer (FIT) of the organic phase electrolyte anion by HEWL; adsorption of this anion-HEWL complex; and, on scanning back to lower potentials, desorption of the anion-protein complex[3, 17]. These proposed phenomena correspond to characteristic features in the cyclic voltammetry. The aim of the work reported here was to determine whether formation of a refractive index-matched ITIES enabled similar electrochemical behaviour of HEWL as obtained at an unmodified ITIES. By modifying a typical nonemulsified ITIES system incrementally with glycerol, it is possible map the observed electrochemical response to changes in the composition. The results presented form a foundation for future development of refractive index matched emulsified-ITIES systems, which use glycerol to render the interface isorefractive.

\section{Experimental}

Voltammetry was conducted at a microporous silicon supported micro-ITIES array[19] in which the membrane consisted of eight hexagonally arranged pores of radius $26.6 \mu \mathrm{m}$. The aqueous phase was modified with different concentrations (wt\%) of glycerol and contained $\mathrm{HCl}(10 \mathrm{mM})$ with either tetraethylammonium $\left(\mathrm{TEA}^{+}\right)$or $\mathrm{HEWL}$ as the target analyte. The organic phase was bis(triphenylphosphoranylidene)ammonium tetrakis-(4-chlorophenyl) borate (BTPPATPBCl) $(10 \mathrm{mM})[20]$ in 1,6-dichlorohexane $(1,6 \mathrm{DCH})$ (spectrophotometric grade $99.5 \%$ ) gelled with polyvinylchloride $(10 \% \mathrm{w} / \mathrm{v})$. Electrochemistry conducted at the glassy carbon disk electrode (GCE) ( $3 \mathrm{~mm}$ diameter) was that of three redox couples prepared from hexaammineruthenium(III) chloride, potassium hexacyanoferrate(II) trihydrate and ferrocenecarboxylic acid (FCA) (Alfa Aesar), where all were dissolved in potassium chloride $(0.1 \mathrm{M}$ in water) at $0.001 \mathrm{M}$ with the exception of FCA, which was diluted by a factor 10 times from a saturated aqueous solution $(0.1 \mathrm{M} \mathrm{KCl})$. All materials were purchased from Sigma Aldrich, Australia, and used as received, unless otherwise stated.

Electrochemical measurements were conducted using an AUTOLAB PGSTAT302N potentiostat (Metrohm, The Netherlands). The cell was operated in two-electrode mode where the reference electrodes in each phase were $\mathrm{Ag} / \mathrm{AgCl}$, connected via an organic reference solution in the case of the organic phase, and also served as the counter electrodes. The ITIES was modified by addition of up to $80 \mathrm{wt} \%$ glycerol, which is representative of the solvent conditions required to render the constituent phases isorefractive[11]. 


\section{Results and Discussion}

The transfer potential at the ITIES represents the Gibbs free energy of transfer for a particular species and is dependent on the difference in solvation energy of that species in either phase[21]. Furthermore, the potential at which any response is observed is directly related the Gibbs free energy required to drive that process. With this understanding, it becomes instructive to examine how the position of a particular response on the potential scale changes with respect to modification of the ITIES system composition. Keeping in mind that the purpose of pursuing the refractive index-match ITIES system is to investigate the nature of protein at the electrified ITIES, it is important to understand how the interfacial interaction with HEWL responds to an increasingly glycerol rich environment.
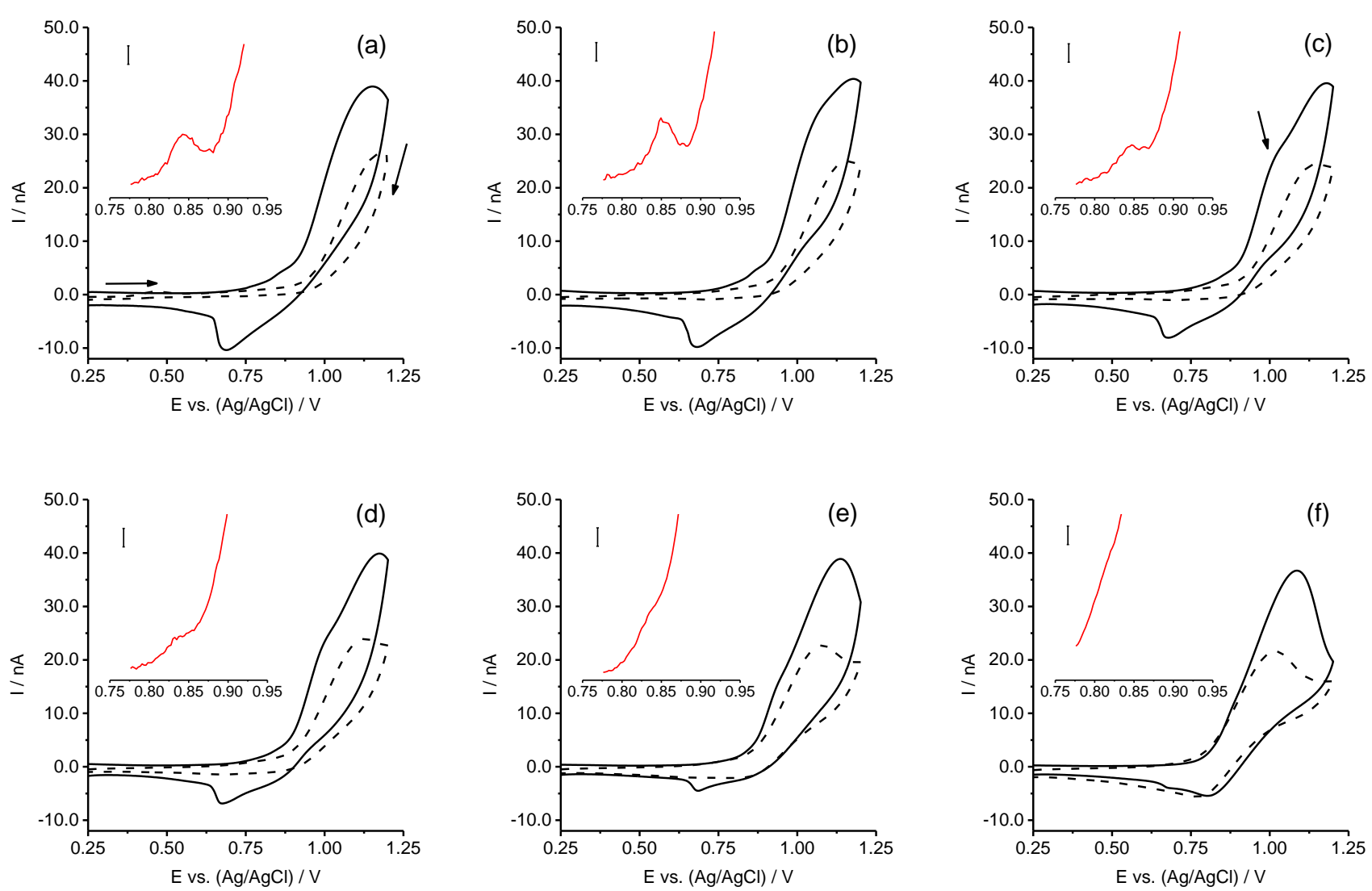

Figure 1. Cyclic voltammograms showing the electrochemical response of HEWL $(20 \mu \mathrm{M})$ at various glycerol concentrations (solid line) and in the absence of HEWL (dashed line). 
Glycerol proportion is varied through (a) 0, (b) 20, (c) 30, (d) 40, (e) 60, (f) 80 wt\%. Sweep rate: $5 \mathrm{mV} \mathrm{s}^{-1}$. The inset for each concentration shows the first derivative plot of current for the region of the forward scan exhibiting the HEWL pre-peak. The scale bar is associated with the inset derivative plots and defines $10 \mathrm{nA} \mathrm{s}{ }^{-1}$. The horizontal axis on both the inset and main plots are of the same units, as indicated for the main plots. Note that the vertical inflection immediately to the right of the peak on the $1^{\text {st }}$ derivative plot represents the pre-peak position. The arrow in (c) indicates the 'main peak'.

\subsection{HEWL at the glycerol modified interface}

The aqueous phase is prepared containing HEWL $(20 \mu \mathrm{M})$, which is considered fully protonated in the presence of $\mathrm{HCl}(10 \mathrm{mM})[3]$. Figure 1(a) shows the typical cyclic voltammetry response for HEWL at the unmodified micro-ITIES array. Several key features in the voltammetry have previously been associated with mechanistic steps[3, 17, 22]. The pre-peak (Figure 1a, forward scan, $0.86 \mathrm{~V}$ ) is associated with adsorption of a HEWL/TPBCl- complex to the interface. This complex, thought to form by complexation of the initially transferred $\mathrm{TPBCl}^{-}$ion(s) with protein near the interface, then adsorbs at the interface. The main peak (indicated in Figure 1c, forward scan, $1.05 \mathrm{~V}$ ) is proposed to be the result of organic phase electrolyte anion transfer facilitated by HEWL in a FIT mechanism. The main-peak and backgroundelectrolyte transfer peak, appear severely convolved for the 0 and 20 wt\% cases (Figure $1 \mathrm{a}$ and $1 \mathrm{~b}$ ). Finally, the desorption-peak (Figure $1 \mathrm{a}$, reverse scan, $0.66 \mathrm{~V}$ ) is associated with desorption of the HEWL/TPBCl- complex from the interface (Figure 1 , reverse scan). This desorption process may involve the decomplexation of the adsorbed species and the subsequent back-transfer of the organic phase anion, which provides the charge transfer step employed in detection of proteins by this approach[23-25], or simply the desorption of the complex to the bulk aqueous phase. The shape and position of the reverse peak remain constant with respect to glycerol concentration, while the peak area decreases gradually with increasing glycerol content. This behaviour suggests that the reverse peak observed for high percentage glycerol systems is still representative of the same desorption process observed for the glycerol-free systems presented here, and reported previously [3, 17, 22]. The decreasing peak area is discussed in more detail below. 
Several changes to the shape of the voltammetry result from modifying the aqueous phase with glycerol. Below $40 \mathrm{wt} \%$ glycerol, the typical pre-peak is apparent, however it does not appear to persist for the glycerol rich systems above $40 \mathrm{wt} \%$ (Figure 1, insets). This is not particularly instructive by itself as there could be a number of explanations, convolution for example. However, the desorption peak on the reverse scan persists for all six glycerol concentrations examined. If the proposed mechanism remains operative, this indicates that adsorption is still occurring despite the absence of the associated pre-peak, in line with results of Day et al.[11] which demonstrated the ability of lysozyme to adsorb at an oil/waterglycerol interface where the glycerol concentration was ca. 80 wt\%. Accordingly, the adsorption of lysozyme is occurring in a way that is comparable to that observed in conventional ITIES systems, but the pre-peak is absent.

Notably, the desorption peak has a significantly larger area than the pre-peak, inline with previous studies which explain this phenomena by means of a secondary adsorption step [3, 17], and appears to decrease in area with increasing glycerol content (Table 1). Typically, the desorption peak-area is proportional to the charge desorbed and reflects the amount of material stripped from the interface during the voltammetric scan. An adsorption dependence on glycerol concentration is important because the density of adsorbed protein could have implications for the quaternary structure of the adsorbed protein layer. Table 1 shows data for the desorption peak charge with respect to glycerol wt\% in the aqueous phase. The dependence of peak charge on glycerol concentration may reflect decreased rates of diffusion during the adsorption process, as the aqueous phase viscosity is increased by addition of glycerol. The rate determining process in this case may be the diffusion of HEWL towards the interface. This is due, in spite of the mass transport advantage delivered by radial diffusion[19], to the higher concentration of TPBCl- present at the interface[3, 17]. The decrease in peak area may also reflect the role of solvation effects in determining adsorption/desorption characteristics. As the onset of the FIT current occurs at progressively lower potentials with respect to the upper-limit of the scan range, the length of time over which the FIT process can occur increases with increasing glycerol concentration for the scans shown in Figure 1. In addition, there is a significant background electrolyte current within this range (See blank scans, Figure 1) which may affect the FIT process and the 
adsorption of protein at the interface. Despite this, an approximately linear $\left(R^{2}=0.971\right)$ decrease in peak area is observed which offers some support to the idea that solvation effects, deactivation of the lysozyme adsorption mechanism due to increasing concentrations of glycerol or, conversely, the deactivation of adsorption sites at the interface, might be the determining factors in this response.

Table 1. Dependence of HEWL desorption peak charge and $\mathrm{TEA}^{+}$half-wave transfer potential from water to $1,6-\mathrm{DCH}$, in cells of varying glycerol content. Potentials are with respect to the experimental reference electrodes.

\begin{tabular}{ccc}
$\begin{array}{c}\text { wt\% glycerol } \\
\text { (aq. phase) }\end{array}$ & $\begin{array}{c}\text { Desorption- } \\
\text { peak charge / } \\
C \times 10^{9}\end{array}$ & $\Delta_{W}^{D C H} \phi_{\frac{1}{2}}\left(T E A^{+}\right) / V$ \\
\hline 0 & 105 & 0.65 \\
20 & 76.4 & 0.62 \\
30 & 50.8 & 0.60 \\
40 & 46.6 & 0.59 \\
60 & 26.8 & 0.55 \\
80 & - & 0.49 \\
\hline
\end{tabular}

It is well established that solvation of bio-macromolecules in non-aqueous or part-aqueous solvent systems can result in significant structural change. Glycerol is a well-established cryosolvent for proteins and has been the subject of numerous studies into polyol stabilisation of protein structure[26]. Recently, Esposito et al. reported minor tertiary structural deformation of HEWL in glycerol rich systems and stabilisation against heat-induced denaturation[16]. It is important to note that even slight changes in protein tertiary structure can result in the deactivation of binding sites and a change in binding affinity; as a result, this can alter the anion binding (FIT) and adsorption reactions crucial to the electrochemical behaviour of HEWL and hence may contribute to the observed responses. Additionally, Spinozzi et al. elucidated the nature of lysozyme solvation under the same glycerol rich conditions as used here[27]. They demonstrated the preferential hydration of lysozyme which may have implications for the proposed protein-complex adsorption/desorption 
process and protein-anionic ligand binding. Further investigation is required to elucidate the solvent dependent nature of this interaction.

The Gibbs free energies required to drive processes at the interface were compared by examining the shift in potential at which the corresponding voltammetric features were observed. The pre-peak does not appear to shift significantly over the 0-40 wt\% range (Figure 2), however as the feature was not apparent for higher glycerol concentrations, it is not clear whether the pre-peak potential is constant over the entire $\mathbf{0 - 8 0} \mathrm{wt} \%$ range. The reverse 'desorption' peak appears to remain constant over the full range of glycerol concentrations examined. This indicates that the adsorption/desorption process is not strongly dependent on the nature of its initial solvent environment and that any change in the structure of the interface due to the presence of glycerol does not significantly affect the ability of the protein-anion complex to adsorb or desorb. This is not to say that the structure of a protein-anion complex adsorbate remains constant.

In contrast, the main peak in the HEWL voltammetry shows a significant shift towards lower values with increasing glycerol concentration up to $80 \mathrm{wt} \%$ (Figure 2). This could reflect a change in the binding affinity of HEWL for the organic phase anion, therefore increasing the energetic advantage of the FIT mechanism as glycerol concentration was increased. Shao et al.[28] discussed changes in the Gibbs free energy of transfer for acetylcholine cations at a sucrose-modified ITIES in terms of the relative energies of ion hydration in the modified and unmodified phases. In the same way, the shift in the FIT peak (main-peak) observed in this work may be attributable to a decrease in the hydration energy of the $\mathrm{TPBCl}^{-}$anion in the modified aqueous phase relative to the unmodified aqueous phase. 


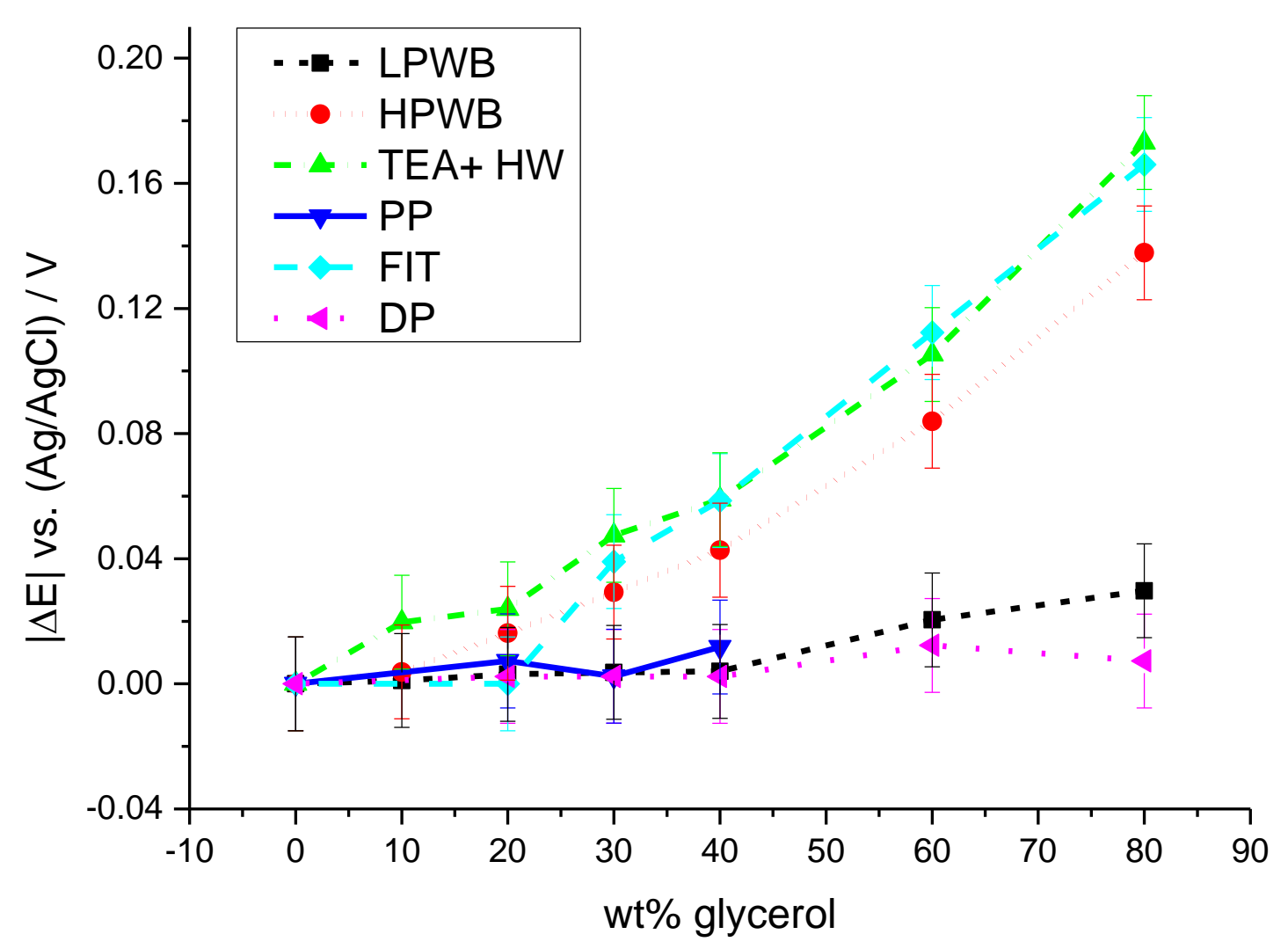

Figure 2. Magnitude of potential shift observed for the lower and higher potential window boundaries (LPWB and HPWB, respectively), $\mathrm{TEA}^{+}$transfer half-wave potential (TEA ${ }^{+} \mathrm{HW}$ ), HEWL pre-peak (PP), HEWL desorption peak (DP) and HEWL main peak (FIT) with respect to glycerol weight percentage in the aqueous phase. All shifts are plotted with respect to the $0 \mathrm{wt} \%$ glycerol response.

The results presented here show that the electrochemical response of HEWL observed under glycerol rich conditions is different to that observed in conventional pure aqueous systems; specifically, a glycerol dependent shift in FIT potential is observed, both the facilitated ion transfer current and the reverse peak charge are diminished, and the pre-peak feature is not observed for the glycerol rich cases $(60$ wt\% and $80 \mathrm{wt} \%)$. 


\subsection{TEA $^{+}$transfer}

In an effort to understand these differences, the well-established electrochemistry of tetraalkylammonium-ion transfer was examined under the same conditions at the glycerol-modified micro-ITIES array. TEA ${ }^{+}$is added to the glycerol modified aqueous phase of the ITIES cell and its electrically driven transfer across the interface is used to probe the nature of the system.

The potential window is the region within which applied potential across the interface is of inadequate magnitude to drive significant background electrolyte transfer current. It follows that the potential window should be dependent on the chemical nature of either phase as far as it effects the solubility of background electrolyte ions[21]. In the absence of a standardized method for potential window measurement, we defined the potential window boundary to be $0.8 \mathrm{nA}$ above or below the existing background current. We observed a significant decrease in potential window (Figure 2) due in large part to a negative shift at the higher potential boundary of the potential window. This boundary is associated with the transfer of $\mathrm{H}^{+}$ions from the aqueous phase to the organic phase and of TPBCl- ions in the opposite direction. The result is rationalised in terms of the increased lipophilicity of the aqueous phase, although it is unclear from the data whether a shift occurred for the chloride/BTPPA ${ }^{+}$transfer, which is represented by the lower potential window boundary. The chloride ion is expected to exhibit a lower magnitude shift than that of the $\mathrm{H}^{+}$ion from primary considerations of the Born model, which dictates that the solvation energy of an ion is inversely proportional to its effective radius[29]. The key limitations of the Born model in this application are that it does not consider specific intermolecular electrostatic interactions, which might influence the solvation energy of an ion such as BTPPA ${ }^{+}$, and it considers the transferring charged species to be spherical, which is not applicable in the case of the BTPPA ${ }^{+}$ion. It is therefore not instructive to consider the solvation energy of $\mathrm{BTPPA}^{+}$in this way.

The transfer of background electrolyte results in a significant current response in the potential region above $0.75 \mathrm{~V}$ on the forward scan (see blanks scans, Figure 1). Interestingly, no obvious reverse process is observed for the electrolyte transfer in systems with low glycerol concentrations, whereas systems with glycerol content greater than $20 \mathrm{wt} \%$ exhibit a reverse current feature in the same region. The peak which occurs beyond the defined upper potential window boundary also shifts to 
lower potentials and is attributed to the simple transfer of $\mathrm{TPBCl}^{-}$ions across the interface on the basis that the observed current response shows a peak shaped profile, indicating linear diffusion; contrarily, radial diffusion is expected for $\mathrm{H}^{+}$ transfer from the aqueous phase[19]. It follows that the current feature observed on the reverse scan of the voltammetry in the absence of lysozyme is the back transfer of $\mathrm{TPBCl}^{-}$from the aqueous phase, which is consistent with its increasing distinction as the glycerol content of the aqueous phase is increased; i.e. as the $\mathrm{TPBCl}^{-}$ion is able to transfer at lower potentials, an increased number of $\mathrm{TPBCl}^{-}$ions are transferred; the decreased diffusion coefficient in the aqueous phase results in a more apparent reverse process as the transferred ions are diluted more slowly into the bulk solution. The dynamics of concurrent transfer processes, transfer of background electrolyte and facilitated ion transfer of $\mathrm{TPBCl}^{-}$, were not investigated in this work.

The introduction of glycerol significantly alters the chemical characteristics of the aqueous phase at higher glycerol concentrations. We hypothesise that this change diminishes the difference in solvation energy between the aqueous and organic phase solvent environments and therefore decreases the Gibbs free energy of transfer of the transferring species to a lower energy. This in turn manifests as a decrease in the magnitude of the transfer potential. We observed a similar trend for $\mathrm{TEA}^{+}$transfer (Figure 3 ) at the 1,6-DCH gel/water-glycerol interface for various water-glycerol proportions (Figure 2) to that discussed above for $\mathrm{TPBCl}^{-}$transfer. The magnitude of the shift in $\mathrm{TEA}^{+}$transfer potential also follows a similar trend to that shown for the HEWL FIT potential with respect to glycerol composition (Figure 2 , TEA ${ }^{+} \mathrm{HW}$ and FIT respectively). This result provides evidence that the shift in HEWL FIT potential occurs predominantly due to a change in phase affinity, however contributions from solvation effects, discussed above, cannot be discounted.

The experimental half-wave potential for $\mathrm{TEA}^{+}$transfer with respect to the silver-silver chloride reference electrodes is presented in Table 1 (derived from Figure 3) and is proportional to the Gibbs free energy of transfer for TEA ${ }^{+}$ions. The negative shift in transfer potential observed represents a decrease in the chemical potential energy across the interface, which results from the addition of the less polar glycerol adsolvent to the aqueous phase. 
The increased viscosity of the aqueous phase was shown to affect the diffusion of the analyte (Figure 3 ) towards the interface in agreement with the Stokes-Einstein relation (1)[30] and as expressed by dependence of the steady-state current on the diffusion coefficient in (2)[19].

$$
\begin{aligned}
& D_{i}=\frac{k_{B} T}{6 \pi \eta a} \\
& I=4 z_{i} F D_{i} C_{i} N r
\end{aligned}
$$

In these equations, $D_{i}$ is the diffusion coefficient of the analyte in the given solvent system, $k_{B}$ is Boltzmann's constant, $T$ is the temperature of the solution, $\eta$ is viscosity of the solvent system, $a$ is the effective radius of the analyte, $I$ is the limiting current, $z_{i}$ is the charge number of the analyte, $F$ is the Faraday constant, $C_{i}$ is the concentration of the analyte in the bulk solution, $N$ is the number of independent interfaces and $r$ is the radius of the pores. Hence, a linear dependence of the diffusion controlled current on the inverse viscosity is expected and obtained $\left(R^{2}=0.994\right.$, Figure 3 , inset $)$, indicating that the decrease in current can be attributed to the increase in viscosity with glycerol composition (Figure 3). Application of this analysis to the HEWL desorption peak charge yielded a poorer linear relationship $\left(R^{2}=0.971\right)$ which suggests that diffusion during the adsorption process is a significant factor in determining the magnitude of the desorption response but that additional factors, such as those discussed above, may influence the desorption process. 


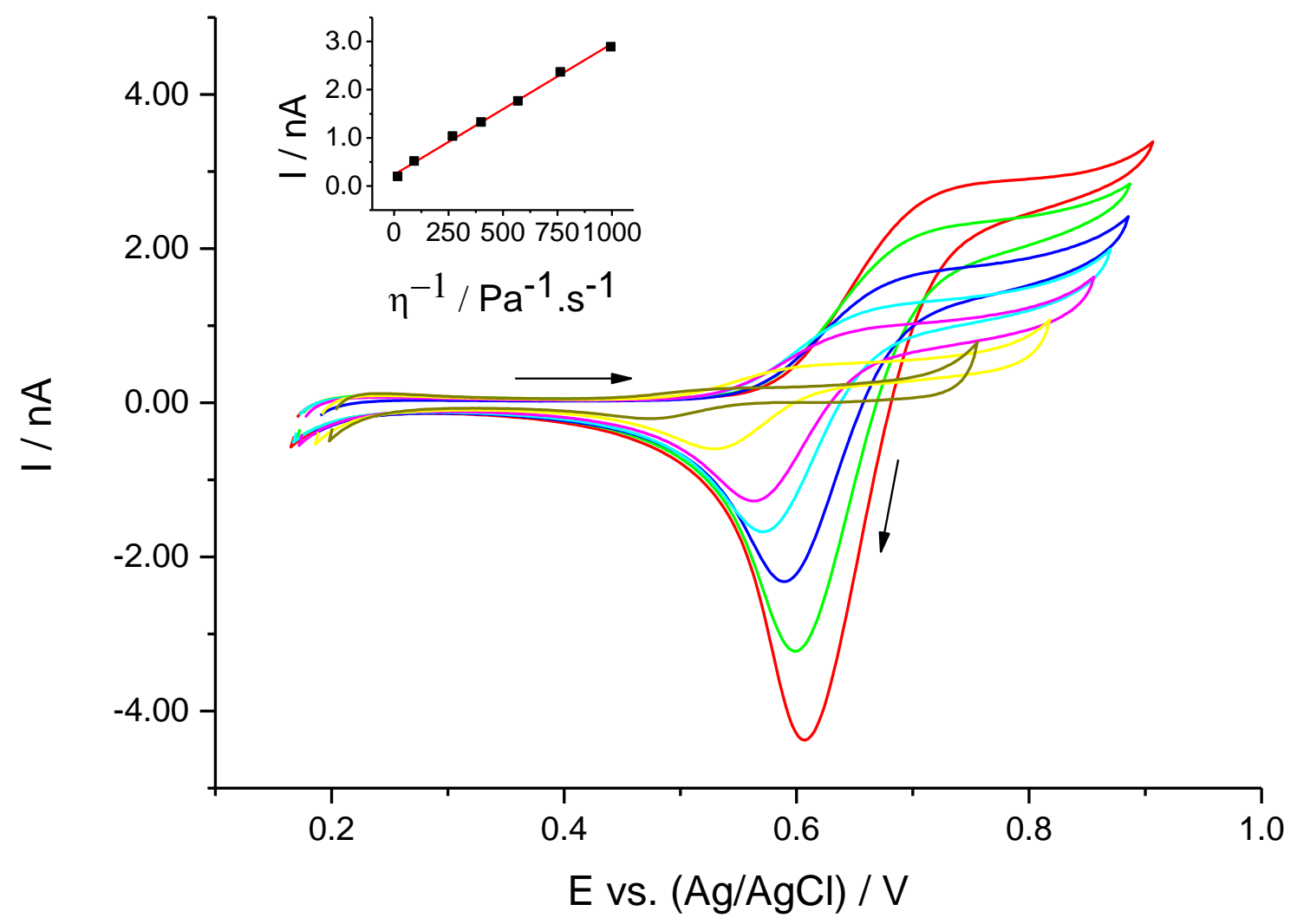

Figure 3. Cyclic voltammetry of $\mathrm{TEA}^{+}$at the glycerol modified micro-ITIES array. Respective glycerol content (wt\%) are denoted by colour: 0 \% (black), $10 \%$ (red), 20 \% (green), 30 \% (blue), $40 \%$ (cyan), $60 \%$ (magenta), $80 \%$ (yellow). The inset plot demonstrates the relationship between current and inverse viscosity, where the viscosity of the solutions is taken to be the viscosity of corresponding water-glycerol mixtures at $20{ }^{\circ} \mathrm{C}$ obtained from [31]. Sweep rate: $5 \mathrm{mV} \mathrm{s}^{-1}$.

\subsection{Reference electrode characterisation}

It is possible that the modification of the aqueous phase with high weightpercentages of glycerol changes the potential of the aqueous phase $\mathrm{Ag} \mid \mathrm{AgCl}$ reference electrode employed, since it was a chloridised-silver wire immersed directly in the aqueous phase. The dependence of the reference electrode potential on electrolyte activity is described by the Nernst equation; a change of ca. $20 \mathrm{mV}$ might result from the diminished relative permittivity of the mixed solvent system and consequent decrease in $\mathrm{Cl}^{-}$activity[32]. 
It was therefore necessary to determine if a shift in the potential resulting from a change in this reference electrode potential was significant. Cyclic voltammetry of three redox couples (Figure 4), ruthenium hexamine, ferrocyanide and FCA, at a glassy-carbon disc electrode was conducted, employing a $\mathrm{Ag} / \mathrm{AgCl}$ reference electrode prepared in the same way as that used in the micro-ITIES array cell. Modification of the aqueous electrolytes with glycerol did not result in significant potential shifts for the ruthenium hexamine or ferrocyanide cases $\left(\Delta \mathrm{E}_{1 / 2}=0.027 \pm 0.005 \mathrm{~V}\right.$ and $0.010 \pm 0.005 \mathrm{~V}$, respectively, Figure 4). However, a large potential shift $\left(\Delta \mathrm{E}_{1 / 2}=0.119 \pm 0.005 \mathrm{~V}\right.$, Figure 4) was observed for FCA. This shift may be attributable to a solvent dependence of the FCA redox potential although a shift in the reference electrode potential cannot be discounted. Previously reported redox potential dependence on carboxylic acid dissociation [33] might be relevant here as the acidity (pKa) of the carboxylic acid moiety is dependent on the waterglycerol composition[34]. No consistent potential shift was observed across the three redox systems examined; however any consistency in potential shift may be obscured by the dependence of the respective redox processes on the solvent environment. As such these results provide only an indication that the potential associated with the aqueous phase $\mathrm{AgCl}$ interface is not significantly dependent on aqueous-phase glycerol composition in the 0-80 wt\% range. Accordingly, we attribute the aqueousphase composition-dependent shift in observed transfer, adsorption and desorption potentials (Figure 2) to a decrease in the Gibbs energy of the respective processes under the glycerol enriched conditions employed.

The mass transport characteristics of the three redox systems at the glassy carbon disc electrode were analysed by combination of the Randles-Sevcik eq.(3),

$$
I_{p}=2.686 * 10^{5} n^{\frac{3}{2}} A \sqrt{D v} C_{B}
$$

where $I_{p}$ is the peak current, $n$ is the number of electrons transferred, $A$ is the electrode area, $D$ is the diffusion coefficient, $v$ is the scan rate and $C_{B}$ is the bulk concentration of the diffusing solute. The expected dependence of current on the viscosity is shown in plots Fig. 4(d-f), consistent with diffusion-controlled behaviour at a disc electrode. 

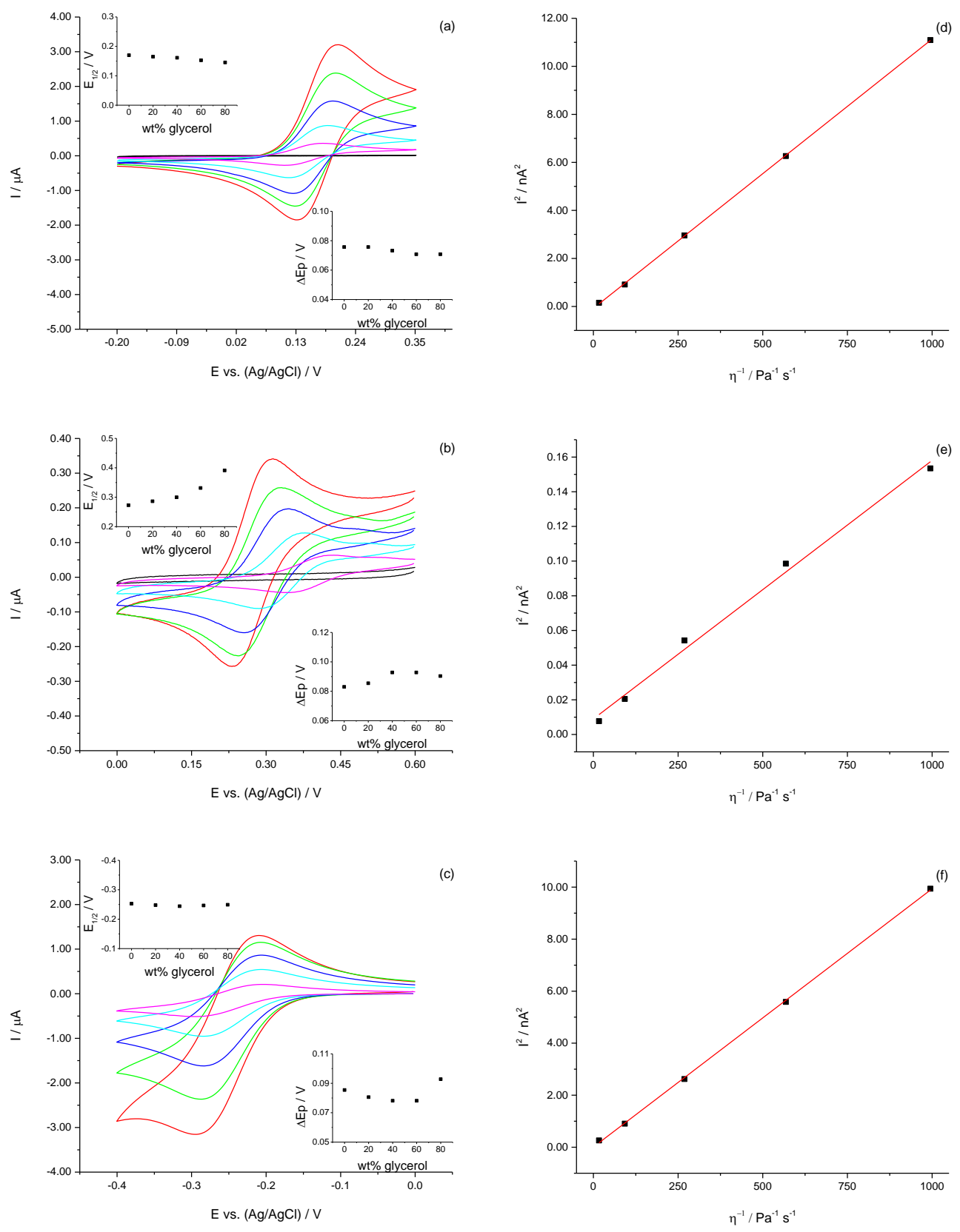

Figure 4. Cyclic voltammetry of ferrocyanide $\left(\mathrm{Fe}(\mathrm{CN})_{6}\right)^{4-}(\mathrm{a}, 1 \mathrm{mM})$, ferrocene carboxylic acid (FCA) (b, 10x diluted saturated solution) and ruthenium hexamine $\left(\left[\mathrm{Ru}\left(\mathrm{NH}_{3}\right)_{6}\right]^{3+}\right)(\mathrm{c}, 1 \mathrm{mM})$ in $0.1 \mathrm{M} \mathrm{KCl}$ solution with various water-glycerol solvent compositons. Inset plots show the half-wave potential and peak-to-peak separation as a function of glycerol concentration. Parts (d) through (f) show the relationship between peak current and viscosity, yielding a linear relationship $\left(R^{2}=0.999\right.$, (d), $\left.\mathrm{Fe}(\mathrm{CN})_{6}\right)^{4-} ; \mathrm{R}^{2}=0.992$, (e), $\mathrm{FCA} ; \mathrm{R}^{2}=0.999$, (f), $\left[\mathrm{Ru}\left(\mathrm{NH}_{3}\right)_{6}\right]^{3+}$ ) between the square of the peak current and inverse viscosity (viscosity values from [31]). 


\section{Conclusion}

The modification of the micro-ITIES array with up to $80 \mathrm{wt} \%$ glycerol added to the aqueous phase resulted in significant changes to the typical electrochemical response of HEWL reported previously. This change was seen in the HEWL response where the facilitated ion transfer peak, corresponding to facilitated TPBCl- transfer, was shown to shift significantly with increasing glycerol concentration. This result reflects a change in chemical potential across the ITIES as a result of the glycerol adsolvent. Interestingly, the potential of peaks associated with adsorption and desorption did not appear to shift significantly with respect to glycerol concentration. This indicates that the energy associated with the adsorptiondesorption process is not strongly influenced by its initial solvent environment. The desorption peak area was observed to decrease with glycerol concentration and exhibited some dependence on viscosity indicating the significance of diminished diffusion, however the contribution of solvation effects cannot be discounted on the basis of the data presented and further investigation is required. Ion transfer voltammetry of $\mathrm{TEA}^{+}$was analysed to provide insight into the nature of the atypical HEWL response. An inverse proportionality between limiting current and viscosity was demonstrated. Furthermore, a negative shift in the $\mathrm{TEA}^{+}$transfer potential demonstrated a decrease in the chemical potential difference across the interface. We also examined the voltammetry of three redox-active compounds at a glassy carbon disc electrode, with the data indicating that the $\mathrm{Ag} / \mathrm{AgCl}$ reference electrode potential was not significantly affected by the presence of glycerol. The results presented demonstrate that the electrochemical signature of HEWL is changed under glycerol-rich conditions, however the electrochemistry which determines this response is similar in both glycerol rich and pure aqueous systems. Accordingly, application of this system to bulk phase spectrophotometric analysis may offer insight into the behaviour of HEWL at the ITIES. Future work should focus on the general nature of protein electrochemistry at glycerol modified interfaces, in particular, the behaviour of less structurally robust proteins, which may exhibit significant instability under the modified conditions presented here.

\section{References}

[1] H.H. Girault, Electrochemistry at liquid-liquid interfaces, in: A.J. Bard \& C.G. Zoski (Eds.), Electroanalytical Chemistry: A Series of Advances, Taylor \& Francis, Boca Raton, FL, 2010. 
[2] D.W.M. Arrigan, Voltammetry of proteins at liquid-liquid interfaces, Annu. Rep. Prog. Chem. C, 109 (2013) 167-188.

[3] M.D. Scanlon, E. Jennings, D.W.M. Arrigan, Electrochemical behaviour of hen-egg-white lysozyme at the polarised water/1,2-dichloroethane interface, Phys. Chem. Chem. Phys., 11 (2009) 2272-2280.

[4] C. Pinholt, R.A. Hartvig, N.J. Medlicott, L. Jorgensen, The importance of interfaces in protein drug delivery - why is protein adsorption of interest in pharmaceutical formulations?, Expert Opinion on Drug Delivery, 8 (2011) 949-964.

[5] A.A. Hyman, C.A. Weber, F. Jülicher, Liquid-Liquid Phase Separation in Biology, Ann. Rev. Cell Dev. Biol., 30 (2014) 39-58.

[6] E. Alvarez de Eulate, L. Qiao, M.D. Scanlon, H.H. Girault, D.W.M. Arrigan, Fingerprinting the tertiary structure of electroadsorbed lysozyme at soft interfaces by electrostatic spray ionization mass spectrometry, Chem. Commun., 50 (2014) 11829-11832.

[7] R.A. Hartvig, M.A. Mendez, M. van de Weert, L. Jorgensen, J. Ostergaard, H.H. Girault, H. Jensen, Interfacial Complexes between a Protein and Lipophilic lons at an Oil-Water Interface, Anal. Chem., 82 (2010) 7699-7705.

[8] A. Barth, Infrared spectroscopy of proteins, Biochim. Biophys. Acta, Bioenergetics, 1767 (2007) 10731101.

[9] S.M. Kelly, T.J. Jess, N.C. Price, How to study proteins by circular dichroism, Biochim. Biophys. Acta, Proteins Proteomics, 1751 (2005) 119-139.

[10] F.A. Husband, M.J. Garrood, A.R. Mackie, G.R. Burnett, P.J. Wilde, Adsorbed protein secondary and tertiary structures by circular dichroism and infrared spectroscopy with refractive index matched emulsions, J. Agr. Food. Chem., 49 (2001) 859-866.

[11] L. Day, J.L. Zhai, M. Xu, N.C. Jones, S.V. Hoffmann, T.J. Wooster, Conformational changes of globular proteins adsorbed at oil-in-water emulsion interfaces examined by Synchrotron Radiation Circular Dichroism, Food Hydrocolloid, 34 (2014) 78-87.

[12] M. A. Rahman, H. Doe, Ion transfer of tetraalkylammonium cations at an interface between frozen aqueous solution and 1,2-dichloroethane, J. Electroanal. Chem., 424 (1997) 159-164.

[13] Y. Tong, P. Sun, Z. Zhang, Y. Shao, Fabrication of agar-gel microelectrodes and their application in the study of ion transfer across the agar-waterl 1,2-dichloroethane interface, J. Electroanal. Chem., 504 (2001) 52-58.

[14] S. Fantini, J. Clohessy, K. Gorgy, F. Fusalba, C. Johans, K. Kontturi, V.J. Cunnane, Influence of the presence of a gel in the water phase on the electrochemical transfer of ionic forms of $\beta$-blockers across a large water|1,2-dichloroethane interface, Eur. J. Pharm. Sci., 18 (2003) 251-257.

[15] H.L.T. Ho, R.A.W. Dryfe, Transport of neutral and ionic solutes: the gel/electrode and gel/electrolyte interfaces, Langmuir, 25 (2009) 12757-12765.

[16] A. Esposito, L. Comez, S. Cinelli, F. Scarponi, G. Onori, Influence of Glycerol on the Structure and Thermal Stability of Lysozyme: A Dynamic Light Scattering and Circular Dichroism Study, J. Phys. Chem. B, 113 (2009) 16420-16424.

[17] M.D. Scanlon, J. Strutwolf, D.W.M. Arrigan, Voltammetric behaviour of biological macromolecules at arrays of aqueous| organogel micro-interfaces, Phys. Chem. Chem. Phys., 12 (2010) 10040-10047.

[18] K. Gekko, Calorimetric study on thermal denaturation of lysozyme in polyol-water mixtures, J. Biochem., 91 (1982) 1197-1204.

[19] R. Zazpe, C. Hibert, J. O'Brien, Y.H. Lanyon, D.W.M. Arrigan, Ion-transfer voltammetry at silicon membrane-based arrays of micro-liquid-liquid interfaces, Lab. Chip, 7 (2007) 1732-1737.

[20] H.J. Lee, P.D. Beattie, B.J. Seddon, M.D. Osborne, H.H. Girault, Amperometric ion sensors based on laser-patterned composite polymer membranes, J. Electroanal. Chem., 440 (1997) 73-82.

[21] Z. Samec, Electrochemistry at the interface between two immiscible electrolyte solutions, Pure Appl. Chem., 76 (2004) 2147-2180.

[22] B.M.B. Felisilda, E. Alvarez de Eulate, D.W.M. Arrigan, Investigation of a solvent-cast organogel to form a liquid-gel microinterface array for electrochemical detection of lysozyme, Anal. Chim. Acta, 893 (2015) 3440. 
[23] E. Alvarez de Eulate, D.W.M. Arrigan, Adsorptive Stripping Voltammetry of Hen-Egg-White-Lysozyme via Adsorption-Desorption at an Array of Liquid-Liquid Microinterfaces, Anal. Chem., 84 (2012) 2505-2511.

[24] S. O'Sullivan, E. Alvarez de Eulate, Y.H. Yuen, E. Helmerhorst, D.W.M. Arrigan, Stripping voltammetric detection of insulin at liquid-liquid microinterfaces in the presence of bovine albumin, Analyst, 138 (2013) 6192-6196.

[25] E. Alvarez de Eulate, L. Serls, D.W.M. Arrigan, Detection of haemoglobin using an adsorption approach at a liquid-liquid microinterface array, Anal. Bioanal. Chem., 405 (2013) 3801-3806.

[26] S. Singh, J. Singh, Effect of polyols on the conformational stability and biological activity of a model protein lysozyme, AAPS PharmSciTech, 4 (2003) 101-109.

[27] F. Spinozzi, M.G. Ortore, R. Sinibaldi, P. Mariani, A. Esposito, S. Cinelli, G. Onori, Microcalorimetric study of thermal unfolding of lysozyme in water/glycerol mixtures: An analysis by solvent exchange model, J. Chem. Phys., 129 (2008) 035101.

[28] Y. Shao, H.H. Girault, Kinetics of the transfer of acetylcholine across the water + sucrose/ 1,2dichloroethane interface, J. Electroanal. Chem., 282 (1991) 59-72.

[29] P.W. Atkins, J. De Paula, Atkins' Physical Chemistry, 9th ed., Oxford University Press, Oxford, 2010. [30] P. Atkins, J. De Paula, Atkins' Physical Chemistry, $8^{\text {th }}$ ed., WH Freeman and Company, New York, 2006. [31] J.B. Segur, H.E. Oberstar, Viscosity of Glycerol and Its Aqueous Solutions, Ind. Eng. Chem., 43 (1951) 2117-2120.

[32] A.J. Bard, L.R. Faulkner, Electrochemical Methods: Fundamentals and Applications, 2nd ed., Wiley, New York, 2001.

[33] G. Desantis, L. Fabbrizzi, M. Licchelli, P. Pallavicini, Controlling the Acidity of the Carboxylic Group by a Ferrocene Based Redox Switch, Inorg. Chim. Acta, 225 (1994) 239-244.

[34] J.T. Rubino, W.S. Berryhill, Effects of solvent polarity on the acid dissociation constants of benzoic acids, J. Pharm. Sci., 75 (1986) 182-186. 